\title{
Superficial Temporal Artery-Sparing Mini-Pterional Approach for Cerebral Aneurysm Surgery
}

\author{
Jun-Young Ahn, M.D., Sung-Tae Kim, M.D., Ki-Chang Yi, M.D., Won-Hee Lee, M.D., Ph.D., Sung Hwa Paeng M.D., Ph.D., \\ Young-Gyun Jeong, M.D., Ph.D. \\ Department of Neurosurgery, Busan Paik Hospital, Inje University, College of Medicine, Busan, Korea
}

Objective : The purposes of this study were to introduce a superficial temporal artery (STA)-sparing mini-pterional approach for the treatment of cerebral aneurysms and review the surgical results of this approach.

Methods : Between June 2010 and December 2015, we performed the STA-sparing mini-pterional approach for 117 patients with 141 unruptured intracranial aneurysms. We analyzed demographic, radiologic, and clinical variables including age, sex, craniotomy size, aneurysm location, height of STA bifurcation, and postoperative complications.

Results : The mean age of patients was 58.4 years. The height of STA bifurcation from the superior border of the zygomatic arch was $20.5 \mathrm{~mm} \pm 10.0$ (standard deviation [SD]). The craniotomy size was $1051.6 \mathrm{~mm}^{2} \pm 206.5$ (SD). Aneurysm neck clipping was possible in all cases. Intradural anterior clinoidectomy was performed in four cases. Contralateral approaches to aneurysms were adopted for four cases. Surgery-related complications occurred in two cases. Permanent morbidity occurred in one case.

Conclusion : Our STA-sparing mini-pterional approach for surgical treatment of cerebral aneurysms is easy to learn and has the advantages of small incision, STA sparing, and a relatively wide surgical field. It may be a good alternative to the conventional pterional approach for treating cerebral aneurysms.

Key Words : Cerebral aneurysm · Pterion · Clipping · Surgery.

\section{INTRODUCTION}

Ever since the classical pterional surgical approach was introduced by Yasagil, it has been adopted worldwide for the clipping of intracerebral aneurysms ${ }^{19)}$. However, this approach requires a wide incision of the temporalis muscle, which can lead to the cosmetic problem of temporalis atrophy ${ }^{13)}$. Because of the development of health medical examination techniques, surgery of unruptured cerebral aneu- rysms is on the rise. It has thus become more important to reduce surgical complications, including cosmetic problems such as this. In order to address this issue, many minimal-invasive surgical techniques have been introduced ${ }^{1,2,4,7,17)}$. However, compared to the classical pterional approach, minimally invasive surgical techniques suffer from some limitations such as narrow surgical fields and a steep learning curve. It is preferable to have techniques that are easy to learn and provide a wide surgical field. Additionally, new surgical ap-

- Received: July 7, 2016 • Accepted: August 22, 2016

- Address for reprints : Young-Gyun Jeong, M.D., Ph.D.

Department of Neurosurgery, Busan Paik Hospital, Inje University College of Medicine, 75 Bokji-ro, Busanjin-gu, Busan 47392, Korea Tel : +82-51-890-6144, Fax : +82-51-898-4244, E-mail: jyk91@inje.ac.kr

This is an Open Access article distributed under the terms of the Creative Commons Attribution Non-Commercial License (http://creativecommons.org/licenses/by-nc/4.0) which permits unrestricted non-commercial use, distribution, and reproduction in any medium, provided the original work is properly cited. 
proaches should optimize the preservation of unaffected structures, especially the superficial temporal artery (STA).

The ideal intracerebral aneurysm clipping technique would be easy for surgeons to learn with a small incision, cause minimal damage to normal structures, and have a relatively wide surgical field. Therefore, we designed the tailored pterional approach for cerebral aneurysm surgery that spares the STA.

The purpose of this study was to introduce our STA- sparing mini-pterional approach for cerebral aneurysm surgery and to review the surgical results of this approach.

\section{MATERIAL AND METHODS}

Between June 2010 and December 2015, we conducted surgeries for 1002 aneurysms in 850 patients at our institute. Of these aneurysms, 415 and 587 were unruptured and ruptured, respectively. This retrospective study was approved by the local ethical committee. Within the stated period, we per- formed the STA-sparing mini-pterional approach for $117 \mathrm{pa}-$ tients with 141 unruptured intracranial aneurysms. All surgical procedures were performed by one senior vascular surgeon. Ruptured aneurysm cases and wrapping cases were excluded. During the same period, the number of coil embolization cases was 682. Among these, the numbers of unruptured and ruptured aneurysms were 459 and 233, respectively.

The height of the STA bifurcation was measured preoperatively in all patients. This was done by calculating the number of cuts from the superior border of the zygomatic arch to the STA bifurcation in 2-mm- sections of brain CT images taken along the orbitomeatal line. Craniotomy size was calculated by the following equation: ([long axis diameter+short axis diameter]/2/2) $2 \times 3.14$. The diameters of the long and short axes were obtained from postoperative CT images. We also analyzed demographic, radiologic, and clinical variables including age, sex, craniotomy size, aneurysm location, height of the STA bifurcation, and postoperative complications.
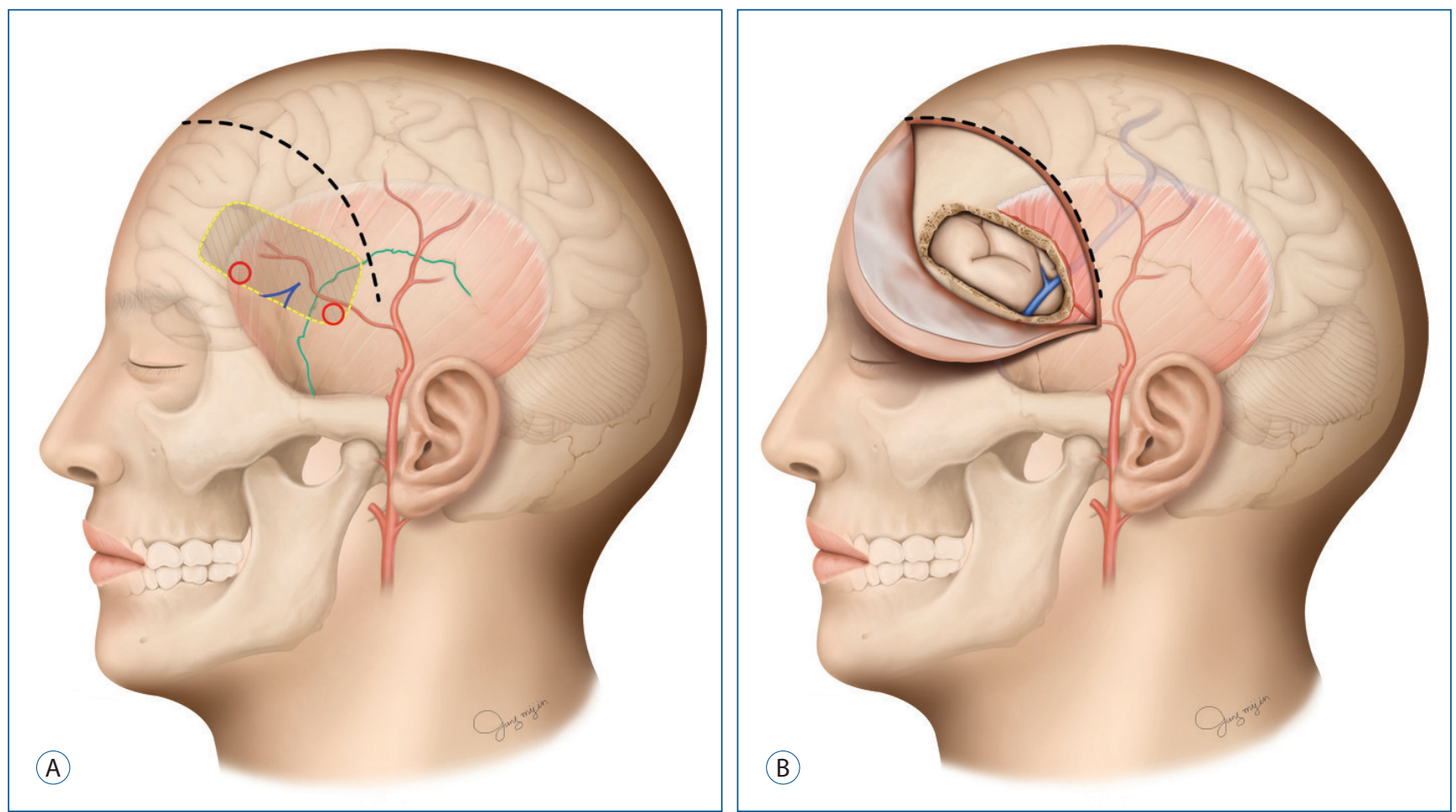

Fig. 1. Schematic drawing of the surgical technique. A : A skin incision is made from the STA bifurcation $\sim 2-3 \mathrm{~cm}$ above the zygomatic arch curvilinearly to the medial hairline (black dotted line). The green line indicates the squamous suture. Red halos indicate burr hole sites. The area surrounded by a yellow dotted line is the craniotomy site. B : After craniotomy, sylvian veins are identified on the temporal base of the field. STA : superficial temporal artery. 


\section{Surgical technique: The STA-sparing mini-pteri- onal approach}

A schematic drawing of this surgical technique is presented in Fig. 1. The patient is placed in the supine position on the operating table. The head is elevated above the heart level to reduce bleeding and is fixed to a three-pronged Mayfield head clamp. The head is rotated 30 to 60 degrees away from the aneurysm side. Then, the neck is extended about 10 degrees to the so-called "malar top" position. After partial hair shaving at the hairline, the STA main trunk and its frontal and parietal branches are measured using micro-Doppler. The skin incision is made just above the STA bifurcation, which is about $2-3 \mathrm{~cm}$ above the zygomatic arch to the medial hairline curvilinearly. If the patient is bald, a median incision can be made in a skin wrinkle. For a wider operative field than that attained with a small incision, sufficient galea dissection is needed. To avoid facial nerve injury, the galea is bluntly dissected along the linea temporalis and STA main trunk with a periosteal elevator. The periostium and superficial temporal fascia should be detached from the base of the frontozygomatic arch to $1 \mathrm{inch}$ above it with a periosteal elevator in order to avoid facial nerve damage. The anterior and superior part of the temporal muscle is cut by diathermy along the linea temporalis. If an extension of the operative field to temporal lobe is required, the posterior part of temporal muscle should be cut. After reflection of the temporal muscle using a fishhook and suture tie, the first burr hole is made at the key hole. The second burr hole is made below the temporal squamous suture line. In the case of elderly patients, another burr hole may be made in the linea temporalis to prevent dural tearing. Craniotomy is performed with a craniotome. The basal area between two burr holes is drilled off with a cutting bur. A craniotomy of about $3 \times 4 \mathrm{~cm}$ is made. If required, extension to the frontal or temporal area of the bone flap can be performed. The sphenoid ridge is drilled using a diamond bur as needed. Dural tacking sutures are used to prevent postoperative epidural hematoma. The dura is opened in the usual fashion with a curvilinear incision and elevated using a suture tie. Sylvian veins are identified on the temporal base of the field using a microscope.
Sylvian dissection ensures a view that is as wide as that of the classical pterional approach.

\section{RESULTS}

Data was collected for a total 117 patients (30 male, $87 \mathrm{fe}$ male; mean age $58.4 \pm 9.89$ years). In these patients, we performed 120 STA-sparing mini-pterional surgeries and clipping of 141 aneurysms. STA-sparing mini-pterional surgery was possible in all patients; this was confirmed by brain CT angiography on postoperative day 7 (Table 1).

The mean craniotomy size was $1051.6 \pm 206.5 \mathrm{~mm}^{2}$. The mean aneurysm size was $3.92 \pm 1.77 \mathrm{~mm}$, and ranged from 1.5 to $10.8 \mathrm{~mm}$. The mean height of the STA bifurcation was 20.5 $\pm 10.0 \mathrm{~mm}$ from the superior border of the zygomatic arch.

The locations of aneurysms were as follows: middle cerebral artery (MCA) bifurcation $(\mathrm{n}=79)$, anterior choroidal artery $(n=13)$, anterior communicating artery $(n=11)$, posterior communicating artery $(n=10), M 1$ segment of the MCA $(n=10)$, A1 segment of the anterior cerebral artery $(n=4)$, internal carotid artery (ICA) bifurcation $(n=4)$, and paraclinoid ICA $(n=10)$. Among the 10 cases of paraclinoid ICA aneurysms, 4 received intradural anterior clinoidectomy.

Clipping multiple aneurysms in a single was necessary in 18 cases. In five cases, three aneurysms were clipped during one craniotomy. Two cases received bilateral craniotomies. Contralateral aneurysm clipping was conducted in four cases (Table 2).

There were two surgical complications. In one, a right

Table 1. Patients' demographic and clinical variables

\begin{tabular}{lc}
\hline Variable & Measurement \\
\hline Sex $(M / F)$ & $30 / 87$ \\
Age in years (mean \pm SD) & $58.4 \pm 9.89$ \\
Mean craniotomy size, $\mathrm{mm}^{2}$ (mean \pm SD) & $1051.6 \pm 206.5$ \\
STA bifurcation height, $\mathrm{mm}$ (mean \pm SD) & $20.5 \pm 10.0$ \\
Aneurysm size, mm (mean \pm SD; minimum, & $3.92 \pm 1.77 ; 1,10.8$ \\
$\quad$ maximum) & \\
\hline$M$ : male, F : female, SD : standard deviation, STA : superficial temporal &
\end{tabular}


Table 2. Location of aneurysms treated using the superficial temporal artery-sparing mini-pterional approach

\begin{tabular}{lc}
\hline Location of aneurysm & $\mathbf{n}$ \\
\hline ACom & 11 \\
PCom & 10 \\
AChA & 13 \\
MCA bif. & 79 \\
M1 & 10 \\
A1 & 4 \\
ICA (paraclinoid) & $10\left({ }^{*} 4\right)$ \\
ICA bif. & 4 \\
Total & 141 \\
\hline
\end{tabular}

*The number of cases for which intradural anterior clinoidectomy was performed. ACom : anterior communicating artery, PCom : posterior communicating artery, AchA : anterior choroidal artery, MCA : middle cerebral artery, bif. : bifurcation, M1 : first segment of the MCA, A1 : first segment of anterior cerebral artery, ICA : internal carotid artery

MCA perforating artery infarction occurred after clipping a right MCA bifurcation aneurysm. The modified Rankin Scale (mRS) score of this patient was 3 at the time of discharge, and had decreased to 2 at the 6-month follow-up. In the second case, a subdural hematoma occurred after clipping a left MCA bifurcation aneurysm. Apart from a mild headache, the patient had no complaints. This patient's mRS score was 1 at discharge and 0 at the 6-month follow-up.

\section{Case illustration 1}

A 74-year-old man had three unruptured cerebral aneurysms (Fig. 2). One was located in the left MCA bifurcation, and two aneurysms were located in the left paraclinoid ICA. A left side approach was performed. The STA bifurcation was $\sim 32 \mathrm{~mm}$ above the superior border of the zygomatic arch. The skin incision was made just above the STA bifurcation, about $3 \mathrm{~cm}$ above the zygomatic arch to the medial hairline curvilinearly. A mini craniotomy $(3 \times 5 \mathrm{~cm})$ that extended to the temporal side was made. After dural incision, the sylvian vein and frontal lobe were exposed. Clipping of three aneurysms was possible after sylvian dissection and cutting the falciform ligament. The patient recovered without any problems.

\section{Case illustration 2}

A 57-year-old woman had three unruptured cerebral aneurysms (Fig. 3) located in right middle cerebral artery bifurcation, right internal carotid artery bifurcation, and left anterior choroidal artery. A right side approach was performed. STA bifurcation was $\sim 26 \mathrm{~mm}$ above the superior border of the zygomatic arch. After reflection of the temporal muscle, a mini craniotomy $(3 \times 5 \mathrm{~cm})$ that extended to the frontal side was made. After sylvian dissection, the left anterior choroidal artery aneurysm was clipped via the contralateral approach. The other two aneurysms were clipped immediately. The patient recovered without any problems.

\section{DISCUSSION}

After the International Subarachnoid Aneurysm Trial, endovascular treatment became the main surgical method to treat cerebral aneurysms ${ }^{8,11,16)}$. However, depending on the anatomic configuration of the aneurysm, it can be difficult to treat with this approach. This is true for wide neck aneurysms, blood blister-like aneurysms, and giant thrombosed aneurysms. Furthermore, coiling has low long-term durability $^{12)}$. For these reasons, surgical clipping still plays a primary role in aneurysm treatment.

As the treatment of unruptured cerebral aneurysms becomes more common, the qualification of treatment is becoming stricter. It is important to satisfy both objective neurological outcomes and the patient's subjective factors. The risk of scar formation, temporal muscle atrophy, and facial nerve palsy limit the use of surgical clipping. To overcome these problems, minimally invasive surgical techniques have been developed. The primary advantage of these techniques is that they reduce the wound size by using tailored craniotomies.

Minimally invasive surgical techniques are classified into two groups depending on the craniotomy approach. The first approach is "keyhole" craniotomy, which is in turn classified into two groups depending on the approach way to the aneurysm. The first is the "subfrontal approach" to the aneu- 

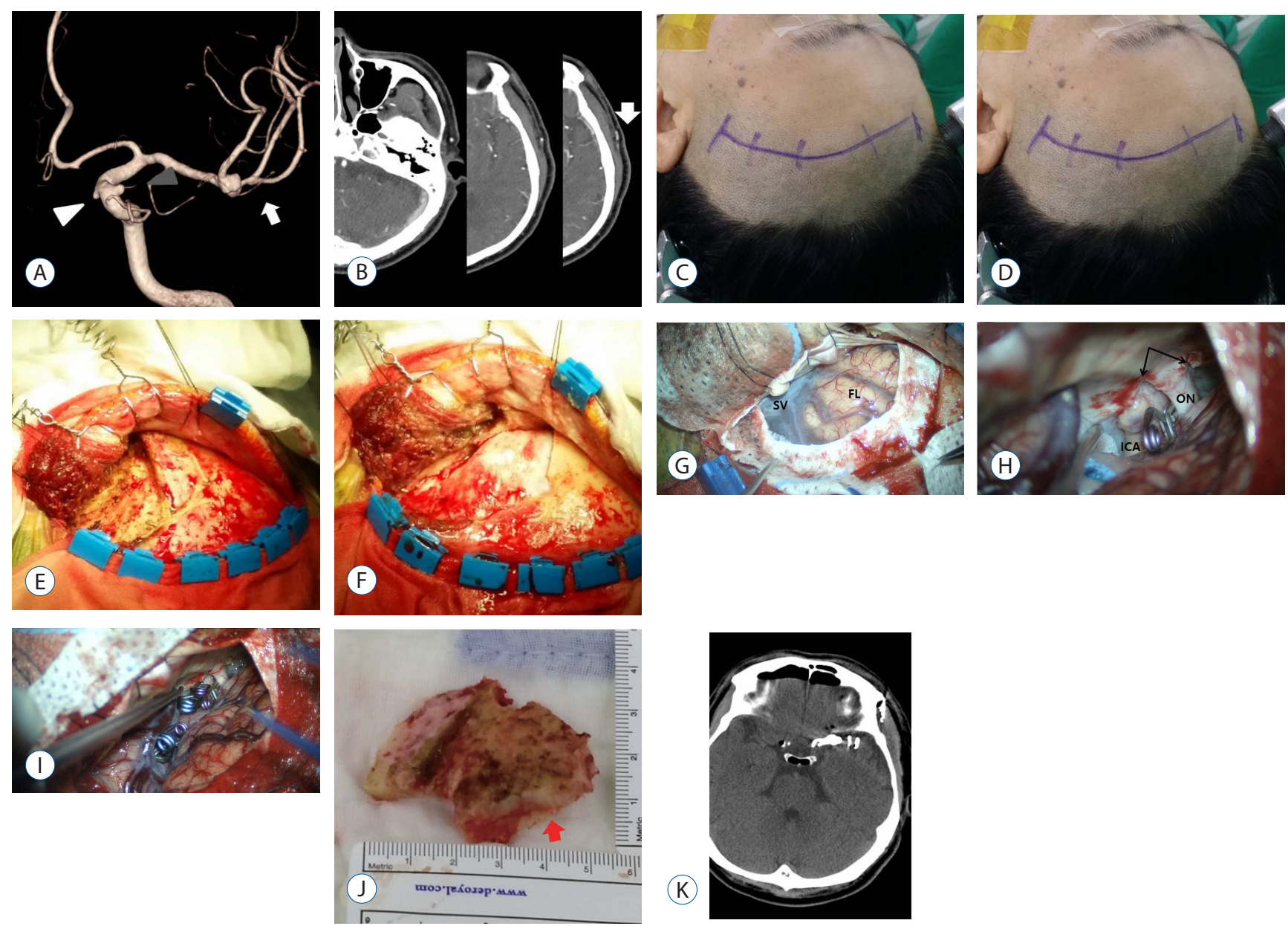

Fig. 2. Case illustration $1 . A$ : White arrows and white and gray arrowheads indicate three aneurysms that were located in the left middle cerebral artery bifurcation and the dorsal and ventral walls of the paraclinoid ICA, respectively, on a 3D cerebral angiography image. B : The white arrow indicates the bifurcation of the STA frontal and parietal branches. C : A skin incision was made from the STA bifurcation ( 3 $\mathrm{cm}$ above the zygomatic arch) to the medial hairline curvilinearly. D : The anterior and superior part of the temporal muscle was cut. E : Reflection of the temporal muscle using fishhook and suture ties. F : Mini craniotomy $(3 \times 5 \mathrm{~cm})$ was performed using a craniotome. $\mathrm{G}$ : The frontal lobe and sylvian fissure as seen under a microscope after dural opening. $\mathrm{H}$ : The internal carotid artery dorsal wall aneurysm was clipped after falciform ligament cutting. I : Multiple clips were seen along the sylvian fissure after clipping. J : The mini craniotomy $(3 \times 5 \mathrm{~cm})$ extended to the temporal side (the red arrow indicates temporally extended craniotomy). $\mathrm{K}$ : Postoperative CT scan showing clips of three aneurysms. ICA : internal carotid artery, STA : superficial temporal artery, CT : computed tomography, FL : frontal lobe, ON : optic nerve, SV: sylvian vein.

rysm $^{5}$. This includes the supraorbital keyhole approach using eyebrow incision ${ }^{4,17}$. The second is the "trans-sylvian approach"14) $^{\text {"14 }}$ that includes the pterional keyhole approach ${ }^{1,18}$. These so-called "keyhole" surgeries have the advantage of a small wound. However, they also have the inherent limitation of a narrow surgical field, leading to several problems including limited use of instruments, difficulty securing the parent artery after premature rupture, and difficulty seeing the back wall of an aneurysm. The second group of minimally invasive surgical techniques is known as the "tailored" pterional approach $^{2,3,6,7,19)}$. The aim of this approach is to re- duce the surgical wound size while maintaining a surgical field that is as wide as possible. However, atrophy of the temporalis muscle can not be resolved ${ }^{6,10,13,18)}$.

Our mini-pterional approach falls into the category of a "tailored" pterional approach, and due to the small skin incision, small temporalis muscle incision, and small craniotomy size, it has a cosmetic advantage over the conventional pterional approach. Accurate craniotomy around the arachnoid dissection point is required to maintain a wide surgical field. Minimal incision of the temporal muscle is important for minimizing temporal muscle atrophy. However, the orienta- 

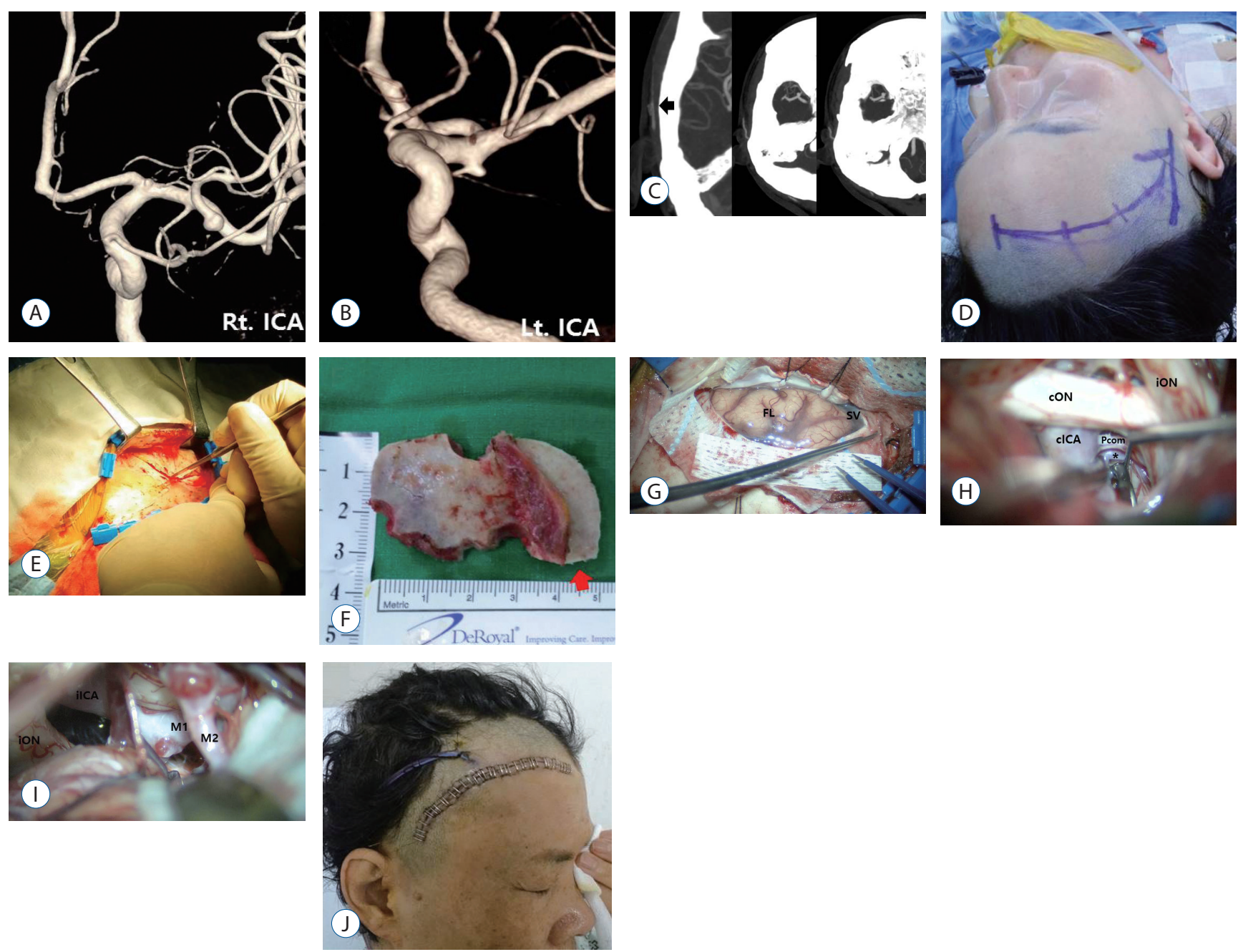

Fig. 3. Case Illustration 2. A and B : 3D image of cerebral angiography showing three aneurysms located in the right middle cerebral artery bifurcation, the right internal carotid artery bifurcation, and the left anterior choroidal artery. C : The black arrow indicates the bifurcation of the STA frontal and parietal branches. D : The skin incision was made curvilinearly from the STA bifurcation $\sim 3 \mathrm{~cm}$ above zygomatic arch to the medial hairline. E : The anterior and superior part of the temporal muscle was cut. F : A mini craniotomy $(3 \times 5 \mathrm{~cm})$ that extended to the frontal side was made (the red arrow indicates frontally extended craniotomy). $\mathrm{G}$ : Microscopic view of the frontal lobe and sylvian fissure after dural opening. $\mathrm{H}$ : Microscopic contralateral view showing the clip at the left anterior choroidal artery. I : Microscopic view of the internal cerebral and middle cerebral artery aneurysms after arachnoid dissection. J : The lateral end of incision, $\sim 2 \mathrm{~cm}$ above the auricle. K : Postoperative CT scan showing clips of three aneurysms. STA : superficial temporal artery, CT : computed tomography, cICA : contralateral internal cerebral artery, CON : contralateral optic nerve, FL : frontal lobe, ilCA : ipsilateral internal cerebral artery, iON : ipsilateral optic nerve, $\mathrm{M}$ : middle cerebral artery, PCom : posterior communicating artery, SV : sylvian vein.

tion of the craniotomy site may be confusing in this situation. The squamous suture acts as an indicator of the sylvian fissure location $^{15)}$. Therefore, the key point of our mini-pterional approach is that squamous suture must be identified, and the burr hole of the temporal side must be made below it. Although it involves a small craniotomy, the accuracy of craniotomy can provide an operative view that is as wide as that of the conventional pterional approach. Furthermore, intradural anterior clinoidectomy and the contralateral ap- proach using this craniotomy were feasible.

The STA bifurcations were found to be, on average, 20.5 $\mathrm{mm}$ above the superior border of the zygomatic arch. This result is similar to previous research ${ }^{9)}$. STA sparing was possible in all surgeries and may confer several advantages such as the reduction of subgaleal hematoma and stimulation of wound healing. Furthermore, the spared STA can provide a solution for unexpected events such as a donor for rescue bypass. Another advantage of our mini-pterional technique is 
that it is easy for neurosurgeons to learn since it is similar to the conventional pterional approach. The bone flap size can be easily adjusted to a frontal or temporal direction in the operative field.

One limitation of this study is that it is retrospective and lacks quantitative analysis of cosmetic factors. Although we need to test this approach in more patients, the evidence presented here supports our view that the STA-sparing minipterional approach is a safe and easy surgical technique.

\section{CONCLUSION}

Our easy-to-learn mini-pterional approach for surgical treatment of cerebral aneurysms has the advantages of a small incision, STA sparing, and a relatively wide surgical field. It may be a good alternative to the conventional pterional approach for treatment of cerebral aneurysm clipping.

\section{References}

1. Cheng WY, Lee HT, Sun MH, Shen CC : A pterion keyhole approach for the treatment of anterior circulation aneurysms. Minim Invasive Neurosurg $49: 257-262,2006$

2. Figueiredo EG, Deshmukh P, Nakaji P, Crusius MU, Crawford N, Spetzler RF, et al. : The minipterional craniotomy: Technical description and anatomic assessment. Neurosurgery 61(5 Suppl 2) : 256-64; discussion 264-265, 2007

3. Figueiredo EG, Deshmukh P, Zabramski JM, Preul MC, Crawford NR, Spetzler RF : The pterional-transsylvian approach: An analytical study. Neurosurgery 62(6 Suppl 3) : 1361-1367, 2008

4. Figueiredo EG, Deshmukh V, Nakaji P, Deshmukh P, Crusius MU, Crawford $N$, et al. : An anatomical evaluation of the mini-supraorbital approach and comparison with standard craniotomies. Neurosurgery 59(4 Suppl 2) : ONS212-220; discussion ONS220, 2006

5. Grand $W$ : Microsurgical anatomy of the proximal middle cerebral artery and the internal carotid artery bifurcation. Neurosurgery $7: 215-218$, 1980

6. Harland SP, Hussein A, Gullan RW : Modification of the standard pterional approach for aneurysms of the anterior circle of willis. Br J Neuro- surg 10 : 149-153; discussion 153, 1996

7. Hernesniemi J, Ishii K, Niemela M, Smrcka M, Kivipelto L, Fujiki M, et al. : Lateral supraorbital approach as an alternative to the classical pterional approach. Acta Neurochir Suppl 94 : 17-21, 2005

8. Jeong HW, Seo JH, Kim ST, Jung CK, Suh SI : Clinical practice guideline for the management of intracranial aneurysms. Neurointervention 9 : 63-71, 2014

9. Kim BS, Jung YJ, Chang CH, Choi BY : The anatomy of the superficial temporal artery in adult koreans using 3-dimensional computed tomographic angiogram: Clinical research. J Cerebrovasc Endovasc Neurosurg $15: 145-151,2013$

10. Miyazawa $T$ : Less invasive reconstruction of the temporalis muscle for pterional craniotomy: Modified procedures. Surg Neurol 50 : 347-351; discussion 351, 1998

11. Molyneux A, Kerr R, Stratton I, Sandercock P, Clarke M, Shrimpton J, et al. : International subarachnoid aneurysm trial (ISAT) of neurosurgical clipping versus endovascular coiling in 2143 patients with ruptured intracranial aneurysms: A randomised trial. Lancet 360 : 1267-1274, 2002

12. Molyneux AJ, Birks J, Clarke A, Sneade M, Kerr RS : The durability of endovascular coiling versus neurosurgical clipping of ruptured cerebral aneurysms: 18 year follow-up of the UK cohort of the international subarachnoid aneurysm trial (ISAT). Lancet 385 : 691-697, 2015

13. Oikawa S, Mizuno M, Muraoka S, Kobayashi S : Retrograde dissection of the temporalis muscle preventing muscle atrophy for pterional craniotomy. technical note. J Neurosurg 84 : 297-299, 1996

14. Pritz MB, Chandler WF : The transsylvian approach to middle cerebral artery bifurcation/trifurcation aneurysms. Surg Neurol 41 : 217-219; discussion 219-220, 1994

15. Rahmah NN, Murata T, Yako T, Horiuchi T, Hongo K : Correlation between squamous suture and sylvian fissure: OSIRIX DICOM viewer study. PLoS One 6 : e18199, 2011

16. Steiner $T$, Juvela $S$, Unterberg $A$, Jung $C$, Forsting $M$, Rinkel $G$, et al. : European stroke organization guidelines for the management of intracranial aneurysms and subarachnoid haemorrhage. Cerebrovasc Dis 35 : 93-112, 2013

17. van Lindert $E$, Perneczky A, Fries $G$, Pierangeli $E$ : The supraorbital keyhole approach to supratentorial aneurysms: concept and technique. Surg Neurol 49 : 481-489; discussion 489-490, 1998

18. Yamahata H, Tokimura H, Tajitsu K, Tsuchiya M, Taniguchi A, Hirabaru $M$, et al. : Efficacy and safety of the pterional keyhole approach for the treatment of anterior circulation aneurysms. Neurosurg Rev 37 : 629636, 2014

19. Yasargil MG, Fox JL: The microsurgical approach to intracranial aneurysms. Surg Neurol 3 : 7-14, 1975 